\title{
EDITORIAL
}

\section{Empirical treatment of uncomplicated cystitis}

Urinary tract infection (UTI) is a frequent disorder in general practice, and encompasses both upper UTI and cystitis (lower UTI). The clinical picture is dependent on age and gender. For young children and the elderly the diagnosis is often uncertain and symptoms and signs may be difficult to interpret $[1,2]$.

Cystitis is a superficial infection in the bladder mucosa, and occurs most commonly in adult women. Uncomplicated cystitis may be defined as acute urinary symptoms (dysuria or urinary frequency) in otherwise healthy non-pregnant women. This condition covers about $95 \%$ of the encounters in general practice due to UTI. Uncomplicated cystitis also accounts for a substantial proportion of antibiotics prescribed in general practice.

Typical symptoms of uncomplicated cystitis are acute, and include dysuria, urge, and urinary frequency. In the absence of vaginal symptoms these symptoms give a $95 \%$ post-symptom probability for uncomplicated cystitis [3]. The diagnosis may then be purely symptom-based, as not even a negative urine dip-stix result may lower the post-test probability sufficiently below a decision-to-treat level [4].

Uncomplicated cystitis is one of the most frequent bacterial infections in general practice, and treatment strategies must as far as possible avoid increase in antibiotic resistance [5]. The condition is self-limited, and symptoms usually disappear in about a week [6]. Complications such as upper UTI are very rare.

In accordance with recent research, several treatment options are available. The options below are valid for adult non-pregnant females with uncomplicated cystitis. Elderly women, pregnant women, men, and children may need a different approach.

- No treatment. Symptoms are self-limited [6] and may be relieved by increased fluid intake, and complications are rare.

- Wait-and-see prescription. This strategy has been shown to result in decreased use of antibiotics for other infections encountered in general practice [7].
- NSAIDs for symptom relief. Ibuprofen equals ciprofloxacin in symptomatic relief [8].

- Three days' treatment with a suitable antimicrobial drug [9].

No treatment is an option if the woman can cope with the symptoms for a few days. NSAIDs have a documentation level of B; one RCT has been published. Results are, however promising, and a few days on NSAIDs may not result in any serious problems. In fact, NSAIDs are sold over the counter in many countries.

The patient should be invited to discuss the available treatment options, and the decision on one of these could be based on mutual agreement. Presenting the options of no treatment or treatment with NSAIDs may make the patient realize that she is not dependent on the doctor when she experiences her next bout of cystitis.

Three days' treatment of uncomplicated cystitis has the same effect as longer courses, but fewer adverse effects [8]. Courses of three days or shorter probably give minor development of bacterial resistance, as shorter treatment courses causes less selection pressure on gut bacteria than longer courses.

Choice of antimicrobial drug should be based on local resistance patterns. Reports on bacterial resistance from laboratories are, however, based on highly selected urine samples from general practice, and will thereby overestimate the actual resistance pattern. Furthermore, most antibacterial drugs reach far higher concentrations in urine than those used in laboratories as MIC levels. A practical rule of thumb may be that an antimicrobial drug may be effectively used in empirical treatment of uncomplicated cystitis when the local resistance level is below $20 \%$.

Nitrofurantoin should be emphasized. This is one of our oldest antimicrobial drugs, and its resistance figures are still favourable all over the world. Trimethoprim is roughly as effective as co-trimoxazole in uncomplicated cystitis, and patients are subject to fewer adverse effects. Because of limited use in many 
countries, its resistance level may be favourable in many regions. Pivmecillinam also mostly shows favourable resistance levels. Quinolones should be reserved for more life-threatening infections, and have no place in the empiric treatment of uncomplicated cystitis.

The listed treatment options are all reasonable. The GP could invite the patient for an informed discussion on what treatment she feels is optimal for her.

\section{Anders Baerheim} Professor

Department of Public Health and Primary Health Care University of Bergen, Norway E-mail: anders.barheim@isf.uib.no

\section{References}

[1] O’Brien K, Stanton N, Edwards A, Hood K, Butler CC. Prevalence of urinary tract infection (UTI) in sequential acutely unwell children presenting in primary care: Exploratory study. Scand J Prim Health Care 2011;29: 19-22.
[2] Rohde N, Löfgren S, Strindhall J, Matussek A, Mölstad S. Cytokines in urine in elderly subjects with acute cystitis and asymptomatic bacteriuria. Scand J Prim Health Care 2009;27:74-9.

[3] Bent S, Nallamothu BK, Simel DL, Fihn SD, Saint S. Does this woman have an acute uncomplicated urinary tract infection? JAMA 2002;287:2701-10.

[4] Bærheim A. Nyrer og urinveier [Kidneys and the urinary tract]. In: Hunskår S, red. Allmennmedisin [Family medicine].Oslo: Gyldendal Norsk Forlag; 2012 (in print).

[5] Arason VA, Sigurdson JA. The problems of antibiotic overuse. Scand J Prim Health Care 2010;28:65-6.

[6] Christiaens TC, De Meyere M, Verschraegen G, Peersman W, Heytens S, De Maeseneer JM. Randomised controlled trial of nitrofurantoin versus placebo in the treatment of uncomplicated urinary tract infection in adult women. Br J Gen Pract 2002;52:729-34.

[7] Høye S, Frich JC, Lindbæk M. Use and feasibility of delayed prescribing for respiratory tract infections: A questionnaire survey. BMC Fam Pract 2011;18:12-34.

[8] Bleidorn J, Gagyor I, Wegscvheider K, Hummers-Pradier E. Symptomatic treatment (ibuprofen) or antibiotics (ciprofloxacin) for urinary tract infection? - Results from a randomized controlled pilot trial. BMC Med 2010;8-30.

[9] Milo G, Katchman EA, Paul M, Christiaens T, Baerheim A, Leibovici L. Duration of antibacterial treatment for uncomplicated urinary tract infection in women. Cochrane Database Syst Rev 2005;2:CD004682. 